\title{
With Copula function analysis of structure dependence relation between Exchange rate of dollars and Deposit rate of Turkey
}

\author{
Ayse Metin Karakas ${ }^{1 *}$, Mine Dogan ${ }^{2}$
}

\begin{abstract}
Objective: In this study, for relation between exchange rate of dollar and deposit rate of Turkey, we used copula function modelling.

Material and Methods: For study, data set that gotten from Turkey central bank in the between 2005-2017 years used.

Results: Recently, rapidly increasing of exchange rate of dollars has been effected deposit rate. In our study, according to data sets, there is a positively relation between exchange rate of dollars and deposit rate. Hence, response of central bank has been showed positively for deposit rate in the increasing of exchange rate of dollars. Throughout study, dependency between exchange rate of dollar and deposit rate obtained positively, namely Kendall Tau ( $\tau=0,751)$ and Spearman's Rho $(\rho=0,912)$. This dependency modelled copula function. For this modelling, Chi-Square test that is Goodness-of-fit used.

Conclusion: As result of this test, for our data set is suitable with parameter Joe copula family which is supported positive tail dependence.

Key Words: Copula function, Deposit rate, Exchange rate of dollars, Goodness-of-fit test Kendall Tau, Spearman's Rho.
\end{abstract}

\section{Introduction}

In recent years, the copula functions have literally be located in finance sector. Although the application of copula in finance is principal in risk management and option pricing, other applications have been suggested. The advantages of using a copula by way of correlation have been modelled dependencies between variables in financial models. Pair wise correlation and the marginal distributions of a random vector is not enough to determine its joint distribution. The copula works by joining together the marginal distributions to form a joint distribution. Thus, the dependence relationship is completely determined by the copula. From a theoretical point of view, copulas let the dependence structure of random variables to be modelled without assumptions on the parametric form In 1959, A. Sklar firstly introduced the general notions of a copula (1981 By. B. Schweizer and E. F. Wolff). Cherubini and Luciano (2001) used Archimedean copulas to examine the tail position of distributions and VaR. Malevergne and Sornette (2003) used a copula representation to redefine the portfolio selection theory. Rosenberg and Schuermann (2006) used the copula modelling to aggregate market risk, credit risk and operational risk. Copula is strong equipment to form multidimensional distributions with given or observed marginals. Such that this is strong equipment in financial modelling, it comes to take the lack of normality in returns and the dependence between of extreme values. (Genest et al., 2009). of the marginal distributions.

Received: 13-03-2017 Accepted 29-03-2017 Available Online: 31-03-2017

1 Bitlis Eren University, Faculty of Science, Dept. of Statistics, Bitlis, TR

* Corresponding Author: Ayse Metin Karakas, E-mail: aysekarakas5767@gmail.com Phone: +90 4342220000 


\section{Material and Methods}

The copula is defined as a $C:[0,1]^{2} \rightarrow[0,1]$ that ensures the limiting conditions

$$
\begin{array}{ll}
\checkmark & C(u, 0)=C(0, u)=0 \text { and } C(u, 1)=C(1, u)=u, \forall u \in[0,1] . \\
\checkmark & \left(u_{1}, u_{2}, v_{1}, v_{2}\right) \in[0,1]^{4}, \text { such that } u_{1} \leq u_{2}, v_{1} \leq v_{2} \\
& C\left(u_{2}, v_{2}\right)-C\left(u_{2}, v_{1}\right)-C\left(u_{1}, v_{2}\right)+C\left(u_{1}, v_{1}\right) \geq 0 .
\end{array}
$$

Ultimately, for twice differentiable and 2-increasing property can be replaced by the condition

$$
c(u, v)=\frac{\partial^{2} C(u, v)}{\partial u \partial v} \geq 0
$$

where $c(u, v)$ is the copula density. In the following, for $n$-uniform random $U_{1}, U_{2}, \ldots, U_{n}$ variables, the joint distribution function $C$ is defined

$$
C\left(u_{1}, u_{2}, \ldots, u_{n}, \theta\right)=P\left(U_{1} \leq u_{1}, U_{2} \leq u_{2}, \ldots U_{n} \leq u_{n}\right)
$$

Here $\theta$ is dependence parameter. $[1,2,3,4,5,7,8,9,10]$

2.1. Sklar Theorem: Let $X$ and $Y$ be random variables with continuous distribution functions $F_{X}$ and $F_{Y}$, with $F_{X}(X)$ and $F_{Y}(Y)$ are uniformly distributed on the interval $[0,1]$. Then, there is a copula such that for all $x, y \in R$,

$$
F_{X Y}(X, Y)=C\left(F_{X}(X), F_{Y}(Y) .\right.
$$

The copula $C$ for $(X, Y)$ is the joint distribution function for the pair $F_{X}(X), F_{Y}(Y)$ provided $F_{X}$ and $F_{Y}$ continuous. $[1,2,3,4,5,7,8,10,11,12,13,14]$

2.2. Archimedean Copula: Let $\varphi$ define a function $\phi:[0,1] \rightarrow[0, \infty]$ which is continuous and provides:

$\checkmark \phi(1)=0, \phi(0)=\infty$.

$\checkmark$ For all $t \in(0,1), \phi^{\prime}(t)<0, \varphi$ is decreasing, for all $t \in(0,1) \varphi^{\prime \prime}(t) \geq 0, \varphi$ is convex.

$\varphi$ has an inverse $\varphi^{-1}:[0, \infty] \rightarrow[0,1]$, which has the same properties out of $\phi^{(-1)}(0)=1$ and $\phi^{(-1)}(\infty)=0$. The Archimedean Copula is defined by

$$
C(u, v)=\phi^{(-1)}[\phi(u)+\phi(v)] .
$$

2.3. Gumbel Copula: This Archimedean copula is defines with the help of generator function $\phi(t)=(-\ln t)^{\theta}$, $\theta \geq 1$

$$
C_{\theta}(u, v)=\exp \left(-\left[(-\ln u)^{\theta}+(-\ln v)^{\theta}\right]^{1 / \theta}\right)
$$


Where $\theta$ is the copula parameter restricted to[ $[1, \infty)$. This copula is asymmetric, with more weight in the right tail. Beside this, it is extreme value copula [12].

2.4. Clayton Copula: This Archimedean copula is defines with the help of generator function $\phi(t)=\frac{t^{-\theta}-1}{\theta}, \theta \in[-1, \infty) /\{0\}$

$$
C_{\theta}(u, v)=\left(u^{-\theta}+v^{-\theta}-1\right) .
$$

Where $\theta$ is the copula parameter restricted to $(0, \infty)$. This copula is also asymmetric, but with more weight in the left tail [12].

2.5. Frank Copula: This Archimedean copula is defines with the help of generator function; $\phi(t)=-\ln \frac{-e^{-\theta t}-1}{e^{-\theta}-1}, \theta \in R /\{0\}$;

$$
C_{\theta}(u, v)=-\frac{1}{\theta} \ln \left(1+\frac{\left(e^{-\theta u}-1\right)\left(e^{-\theta v}-1\right)}{\left(e^{-\theta}-1\right)}\right)
$$

where $\theta$ is the copula parameter restricted to $(0, \infty)[12]$.

2.6. Joe Copula: This Archimedean copula is defines with the help of generator function

$$
C_{\theta}(u, v)=1-\left[(1-u)^{\theta}+(1-v)^{\theta}-\left((1-u)^{\theta}(1-v)^{\theta}\right]^{1 / \theta}\right.
$$

Where $\theta$ is the copula parameter restricted to $[1, \infty]$. This copula family is similar to the Gumbel. The right tail positive dependence is stronger more than Gumbel [12].

2.7. Plackett Copula: This copula function is defines

$$
C(u, v)=\frac{1+(\theta-1)-\sqrt{[1+(\theta-1)(u+v)]^{2}-4 \theta(\theta-1) u v}}{2(\theta-1)} .
$$

Where $\theta$ is the copula parameter restricted to $(0, \infty)[12]$.

\subsection{Measuring Dependence}

2.8.1 Spearman's Rho: Similar to approach of Pearson correlation coefficient, to compute the correlation between the pairs $\left(R_{i}, S_{i}\right)$ of ranks have been used. Thus, Spearman's Rho 


$$
\rho_{n}=\frac{\sum_{i=1}^{n}\left(R_{i}-\bar{R}\right)\left(S_{i}-\bar{S}\right)}{\sqrt{\sum_{i=1}^{n}\left(R_{i}-\bar{R}\right)^{2} \sum_{i=1}^{n}\left(S_{i}-\bar{S}\right)^{2}}} \in[-1,1]
$$

where

$$
\bar{R}=\frac{1}{n} \sum_{i=1}^{n} R_{i}=\frac{n+1}{2}=\frac{1}{n} \sum_{i=1}^{n} S_{i}
$$

write. This coefficient that stated expediently in the form

$$
\rho_{n}=\frac{12}{n(n+1)(n-1)} \sum_{i=1}^{n} R_{i} S_{i}-3 \frac{n+1}{n-1} .
$$

Also, $\rho_{n}$ is asymptotically unbiased estimator of

$$
\rho=12 \int_{[0,1]^{2}} u v d C(u, v)-3=12 \int_{[0,1]^{2}} C(u, v) d u d v-3
$$

where the second equality is obtain. Genest (2007). This statement extended Quesada- Molina (1992)

$$
12 \int_{[0,1]^{2}} u v d C_{n}(u, v)-3=\frac{12}{n} \sum_{i=1}^{n} \frac{R_{i}}{n+1} \frac{S_{i}}{n+1}-3=\frac{n-1}{n+1} \rho_{n}
$$

and $C_{n} \rightarrow C$ as $n \rightarrow \infty$. Here the null hypothesis $H_{0}=C=\Pi$ of independence of $X$ and $Y$, the distribution of $\rho_{n}$ is normal with zero mean and variance $1 /(n-1)$, thus for $H_{0}$ approximate $\alpha=0.05$, $\sqrt{n-1}\left|\rho_{n}\right|>z_{\alpha / 2}=1,96$ Genest (2007).

2.8.2. Kendall Tau: Another measure of dependence is Kendall Tau. This measure based on ranks given by

$$
\tau_{n}=\frac{P_{n}-Q_{n}}{\left(\begin{array}{l}
n \\
2
\end{array}\right)}=\frac{4}{n(n-1)} P_{n}-1
$$

where $P_{n}$ and $Q_{n}$ number of concordant and discordant pairs respectively. Here, $\left(X_{i}, Y_{i}\right),\left(X_{j}, Y_{j}\right)$ pairs are concordant $\left(X_{i}-X_{j}\right)\left(Y_{i}-Y_{j}\right)>0$ and these are disconcordant $\left(X_{i}-X_{j}\right)\left(Y_{i}-Y_{j}\right)<0$.

If $\left(X_{i}-X_{j}\right)\left(Y_{i}-Y_{j}\right)>0$; we can say $\left(R_{i}-R_{j}\right)\left(S_{i}-S_{j}\right)>0 . \tau_{n}$ is function of copula $C_{n}$. As $n \rightarrow \infty$,

$$
\begin{aligned}
& C_{n} \rightarrow C, W=\frac{1}{n} \sum_{j=1}^{n} I_{i j}=\frac{1}{n} \#\left\{j: X_{j} \leq X_{i}, Y_{j} \leq Y_{i}\right\}, \\
& \tau_{n}=4 \frac{n}{n-1} \bar{W}-\frac{n+3}{n-1}=4 \int_{[0,1]^{2}} C(u, v) d C(u, v)-1
\end{aligned}
$$


written. $\tau_{n}$ is asymptotically unbiased estimator of $\tau$ and $\tau_{n}$ is normal with zero mean and variance $2(2 n+5) /\{9 n(n-1)\}$. Here the null hypothesis $H_{0}=C=\Pi$ of independence of $X$ and $Y$, thus for $H_{0}$ approximate $\alpha=0.05, \sqrt{9 n(n-1) / 2(2 n+5)}\left|\tau_{n}\right|>1.96$ Genest (2007).

Table 1: Genrator, Parameter space, Kendall Tau and Spearman Rho values of Special Copula Families

\begin{tabular}{|c|c|c|c|c|}
\hline Family & Generator & Parameter & Kendall Tau & Spearman Rho \\
\hline Gumbel & $\phi(t)=(-\ln t)^{\theta}$ & $\theta \in[1, \infty)$ & $\frac{\theta-1}{\theta}$ & \\
\hline Clayton & $\phi(t)=\frac{t^{-\theta}-1}{\theta}$ & $\theta \in[0, \infty)$ & $\frac{\theta}{\theta+2}$ & \\
\hline Frank & $\phi(t)=-\ln \frac{-e^{-\theta t}-1}{e^{-\theta}-1}$ & $\theta \in(-\infty, \infty)$ & $1-\frac{4}{\theta}\left[1-D_{1}(\theta)\right]$ & $1-\frac{12}{\theta}\left[D_{2}(-\theta)-D_{1}(-\theta)\right]$ \\
\hline Joe & $\varphi(t)=-\ln \left[1-(1-t)^{\theta}\right]$ & $\theta \in[1, \infty)$ & $1+\frac{4}{\theta} D_{J}(\theta)$ & - \\
\hline Placket & - & $\theta \in(0, \infty)$ & - & $\frac{\theta+1}{\theta-1}-\frac{2 \theta \ln \theta}{(\theta-1)^{2}}$ \\
\hline
\end{tabular}

\section{Application}

In this study, we used weekly values of deposit rate and exchange rate of dollars from 30.12.2005 to 13.01.2017 that gotten central bank in Turkey. For our data set, we are to select suitable copula family, we firstly form matrix for observed frequencies of deposit rate and exchange rate of dollars. Table 2 presents $5 \times 5$ matrix of these variables. For the data set, we obtain Kendall Tau 0,751 and Spearman's Rho 0,912. Next step of this study, we are to find the best appropriate copula family and the selected copula family is statistically tested. Best model for our data is find by comparing empirical results.

Table 2: For data observed values

\begin{tabular}{ccccccc} 
& $Y_{1}$ & $Y_{2}$ & $Y_{3}$ & $Y_{4}$ & $Y_{5}$ & Sum \\
\hline$X_{1}$ & 79 & 31 & 5 & 1 & 0 & 116 \\
$X_{2}$ & 27 & 59 & 29 & 0 & 0 & 115 \\
$X_{3}$ & 5 & 22 & 55 & 32 & 2 & 116 \\
$X_{4}$ & 4 & 3 & 26 & 74 & 8 & 115 \\
$X_{5}$ & 1 & 0 & 1 & 8 & 106 & 116 \\
Sum & 116 & 115 & 116 & 115 & 116 & 578 \\
\hline
\end{tabular}




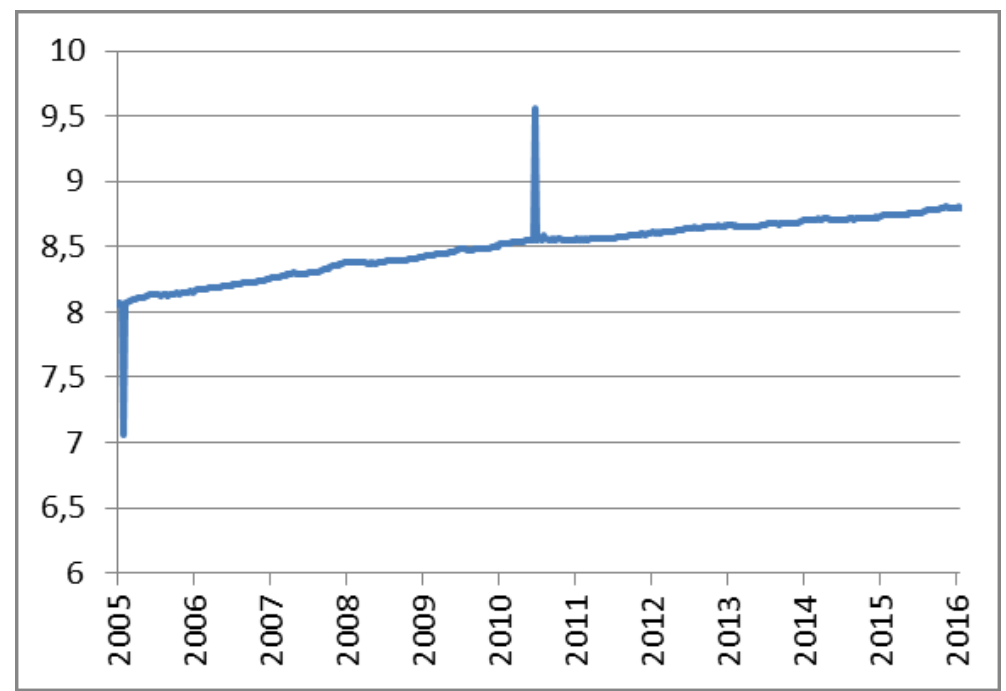

Figure 1: Weekly Deposit Rate of Turkey

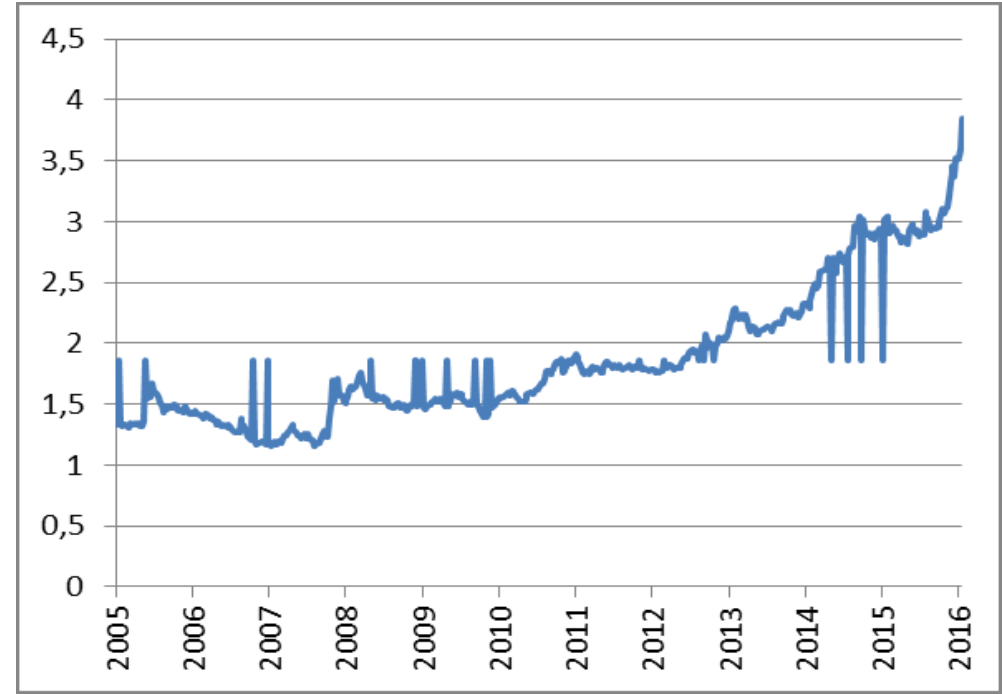

Figure 2: Weekly Exchange Rate of Dollars

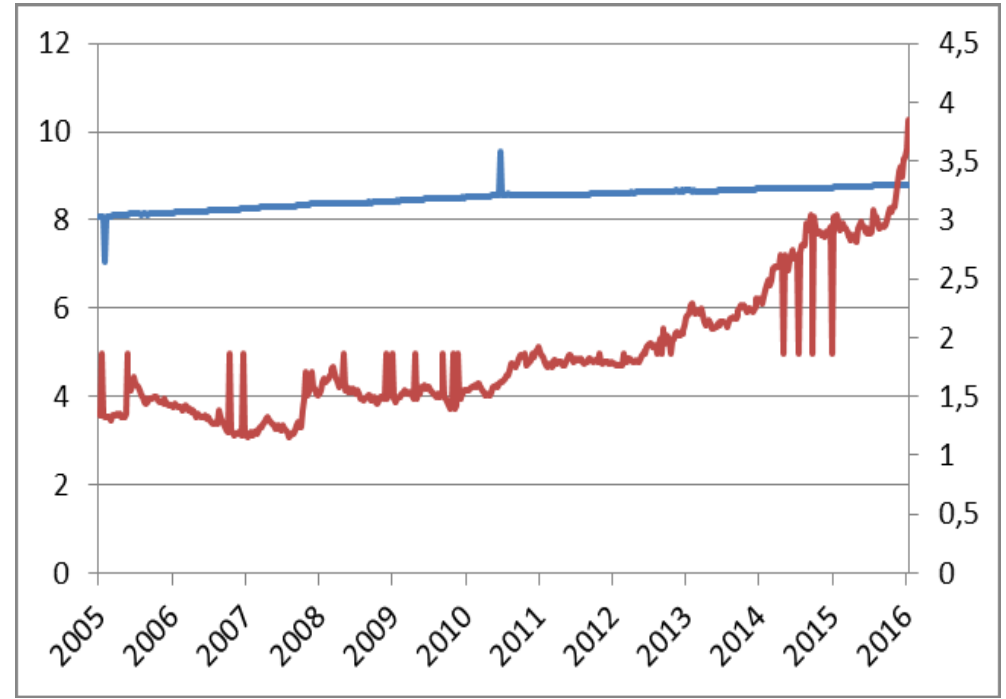

Figure 3: Change of Weekly Deposit Rate of Turkey and Exchange Rate of Dollars 
Table 3: For Data Descriptive Statistics

\begin{tabular}{lllllll} 
Variable & Maximum & Minumum & Std.Dev. & Skewness & Kurtosis & Jarque-Bera \\
\hline Deposit Rate & 9,556957 & 7,064077 & 0,216855 & $-0,64521$ & 5,85234 & 235,6335 \\
Exchange rate of Dollars & 3,849900 & 1,159200 & 0,558684 & 1,08177 & 3,33591 & 115,2508 \\
\hline
\end{tabular}

\subsection{Gumbel Hougaard and Kendall Tau Dependency Estimation}

The connection between Kendall Tau and Gumbel Hougaard copula family is given table 1. From this statement the parameter $\hat{\theta}$ of Gumbel Hougaard copula family is estimated value of 4,016064. The expected frequencies of the $\hat{\theta}$ parameter shows in table 4. Chi-Square value of Gumbel Hougaard copula family calculates as 29,81389. The critical value of Chi-Square test with 16 of freedom and 0,05 confidences level is 26,29623, accordingly Gumbel Hougaard copula family unsuitable for our data set.

Table 4: For Gumbel Hougaard Copula Family Expected Values

\begin{tabular}{lllllll} 
& $Y_{1}$ & $Y_{2}$ & $Y_{3}$ & $Y_{4}$ & $Y_{5}$ & Sum \\
\hline$X_{1}$ & 85 & 26 & 4 & 1 & 0 & 116 \\
$X_{2}$ & 26 & 58 & 27 & 4 & 0 & 115 \\
$X_{3}$ & 4 & 27 & 58 & 25 & 2 & 116 \\
$X_{4}$ & 1 & 4 & 25 & 68 & 17 & 115 \\
$X_{5}$ & 0 & 0 & 2 & 17 & 97 & 116 \\
Sum & 116 & 115 & 116 & 115 & 116 & 578 \\
\hline
\end{tabular}
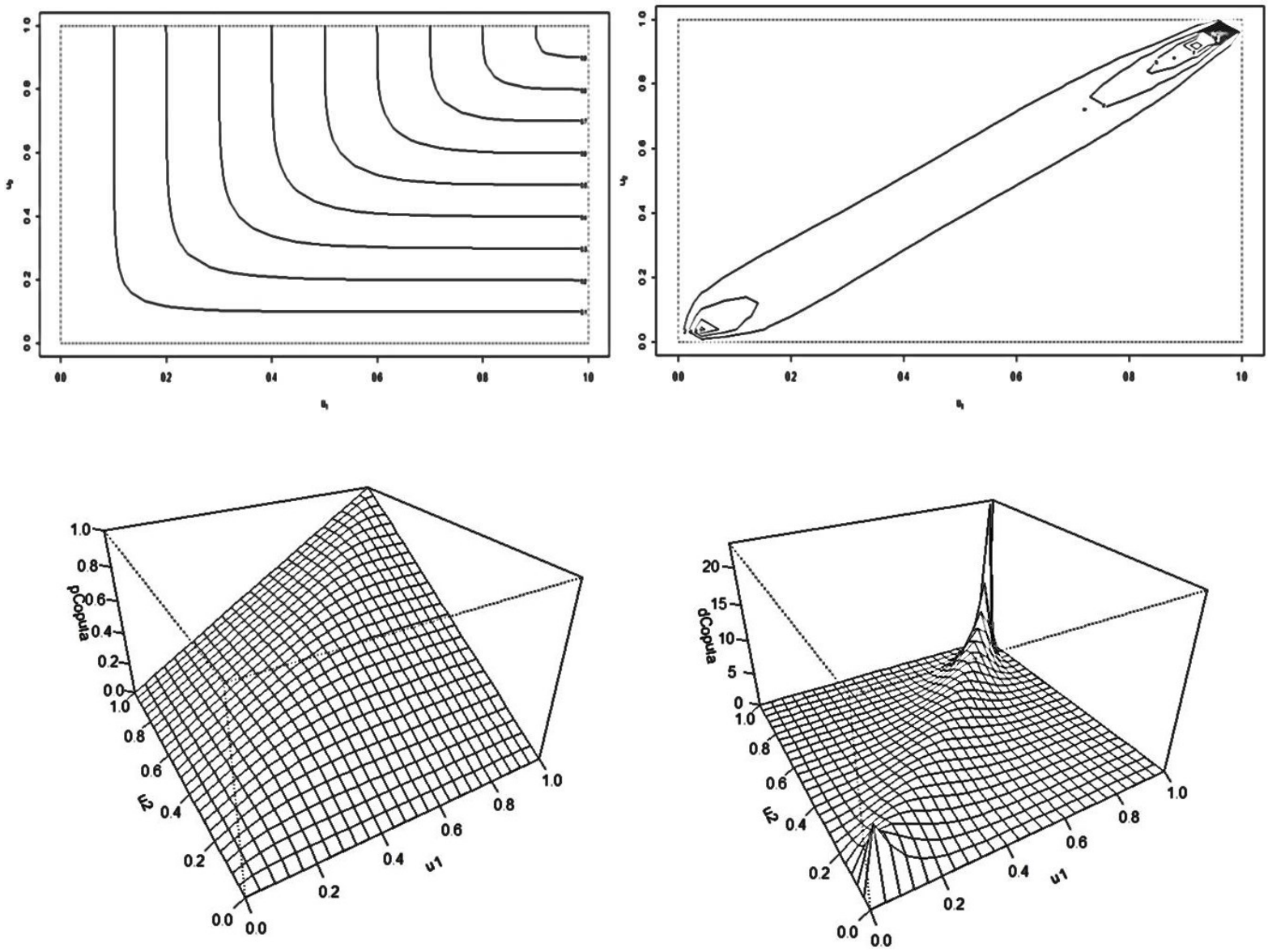
Figure 4: Respectively two and three dimension for Gumbel Hougaard Copula family $\theta=4,016064$, probability and distribution function

\subsection{Clayton and Kendall Tau Dependency Estimation}

The connection between Kendall Tau and Clayton copula family is given table 1. From this statement the parameter $\hat{\theta}$ of Clayton copula family is estimated value of 6.032129. The expected frequencies of the $\hat{\theta}$ parameter shows in table 5. Chi-Square value of Clayton copula family calculates as 163,1352 . The critical value of Chi-Square test with 16 of freedom and 0,05 confidences level is 26,29623, accordingly Clayton copula family unsuitable for our data set.

Table 5: For Clayton Copula Family Expected Values

\begin{tabular}{ccccccc} 
& $Y_{1}$ & $Y_{2}$ & $Y_{3}$ & $Y_{4}$ & $Y_{5}$ & Sum \\
\hline$X_{1}$ & 103 & 12 & 1 & 0 & 0 & 116 \\
$X_{2}$ & 12 & 79 & 21 & 3 & 0 & 115 \\
$X_{3}$ & 1 & 21 & 60 & 27 & 7 & 116 \\
$X_{4}$ & 0 & 3 & 27 & 52 & 33 & 115 \\
$X_{5}$ & 0 & 0 & 7 & 33 & 76 & 116 \\
sum & 116 & 115 & 116 & 115 & 116 & 578 \\
\hline
\end{tabular}
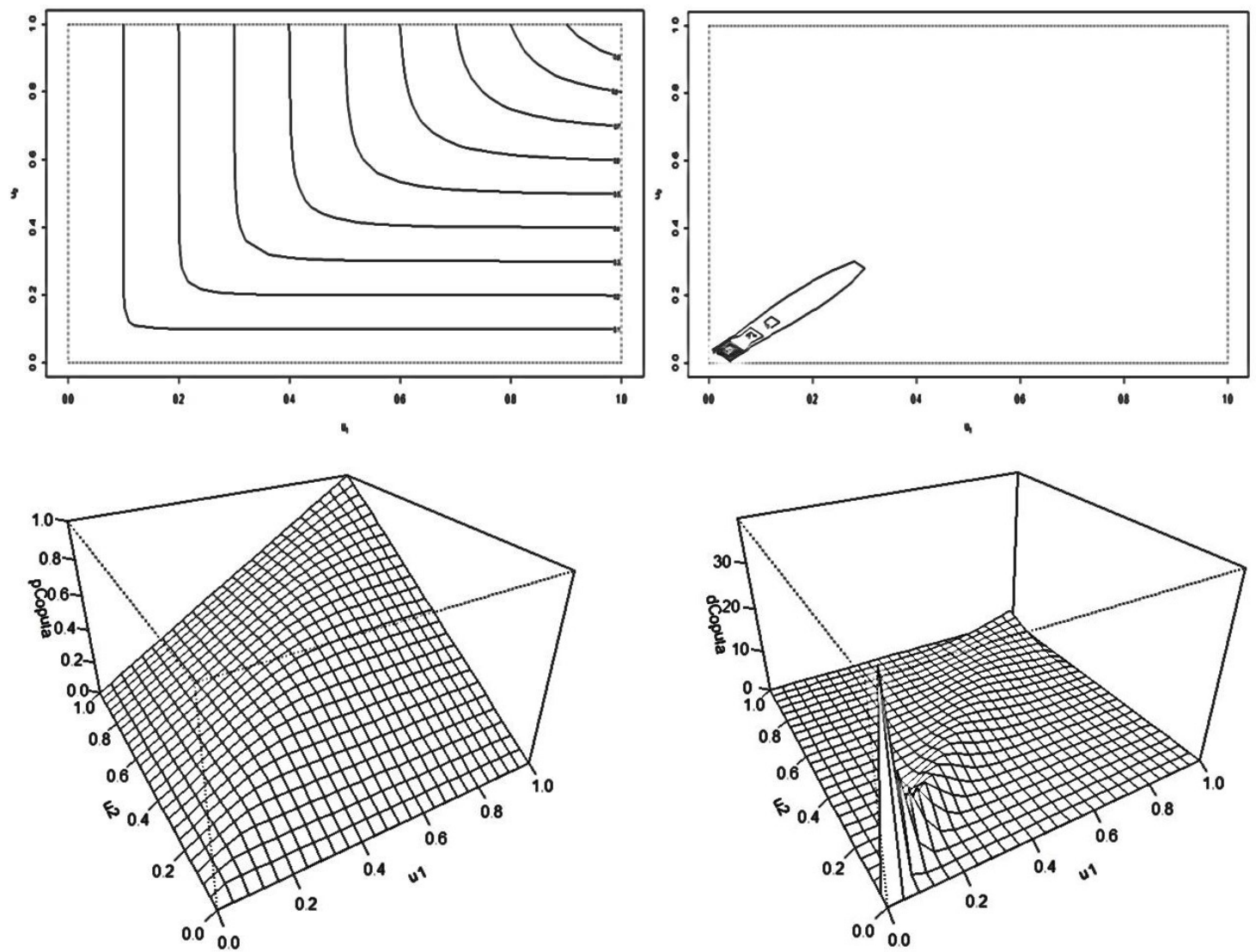

Figure 5: Respectively two and three dimension for Clayton Copula family $\theta=6,032129$, probability and distribution function 


\subsection{Frank and Kendall Tau Dependency Estimation}

The connection between Kendall Tau and Frank copula family is given table 1. From this statement the parameter $\hat{\theta}$ of Frank copula family is estimated value of 14,20389 The expected frequencies of the $\hat{\theta}$ parameter shows in table 6. Chi-Square value of Frank copula family calculates as 61,01967. The critical value of Chi-Square test with 16 of freedom and 0,05 confidences level is 26,29623, accordingly Frank copula family unsuitable for our data set.

Table 6: For Frank Copula Family Expected Values

\begin{tabular}{|c|c|c|c|c|c|c|}
\hline & $Y_{1}$ & $Y_{2}$ & $Y_{3}$ & $Y_{4}$ & $Y_{5}$ & Sum \\
\hline$X_{1}$ & 89 & 24 & 2 & 1 & 0 & 116 \\
\hline$X_{2}$ & 24 & 66 & 24 & 1 & 0 & 115 \\
\hline$X_{3}$ & 2 & 24 & 64 & 24 & 2 & 116 \\
\hline$X_{4}$ & 1 & 1 & 24 & 64 & 25 & 115 \\
\hline$X_{5}$ & 0 & 0 & 2 & 25 & 89 & 116 \\
\hline sum & 116 & 115 & 116 & 115 & 116 & 578 \\
\hline
\end{tabular}
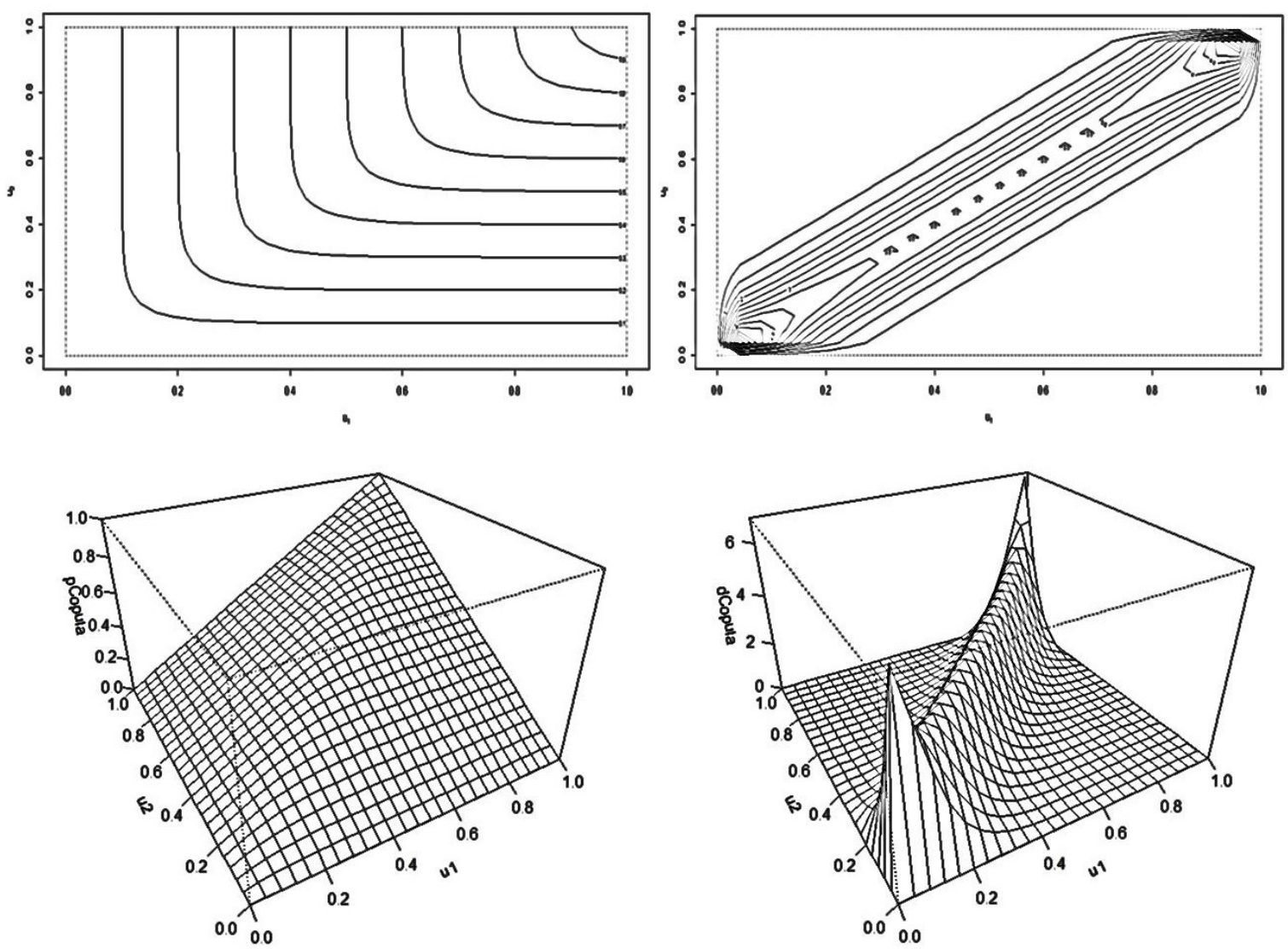

Figure 6: Respectively two and three dimension for Frank Copula family $\theta=14,20389$, probability and distribution function

\subsection{Joe and Kendall Tau Dependency Estimation}

The connection between Kendall Tau and Joe copula family is given table 1. From this statement the parameter $\hat{\theta}$ of Joe copula family is estimated value of 6,8142 The expected frequencies of the $\hat{\theta}$ parameter shows in table 6. Chi-Square value of Joe copula family calculates as 24,26596 . The critical value of Chi-Square test with 16 of freedom and 0,05 confidences level is 26,29623, accordingly Joe copula family suitable for our data set. 
Table 7: For Joe Copula Family Expected Values

\begin{tabular}{ccccccc} 
& $Y_{1}$ & $Y_{2}$ & $Y_{3}$ & $Y_{4}$ & $Y_{5}$ & Sum \\
\hline$X_{1}$ & 75 & 33 & 7 & 1 & 0 & 116 \\
$X_{2}$ & 33 & 54 & 26 & 2 & 0 & 115 \\
$X_{3}$ & 7 & 26 & 61 & 22 & 0 & 116 \\
$X_{4}$ & 1 & 2 & 22 & 78 & 12 & 115 \\
$X_{5}$ & 0 & 0 & 0 & 12 & 104 & 116 \\
sum & 116 & 115 & 116 & 115 & 116 & 578 \\
\hline
\end{tabular}
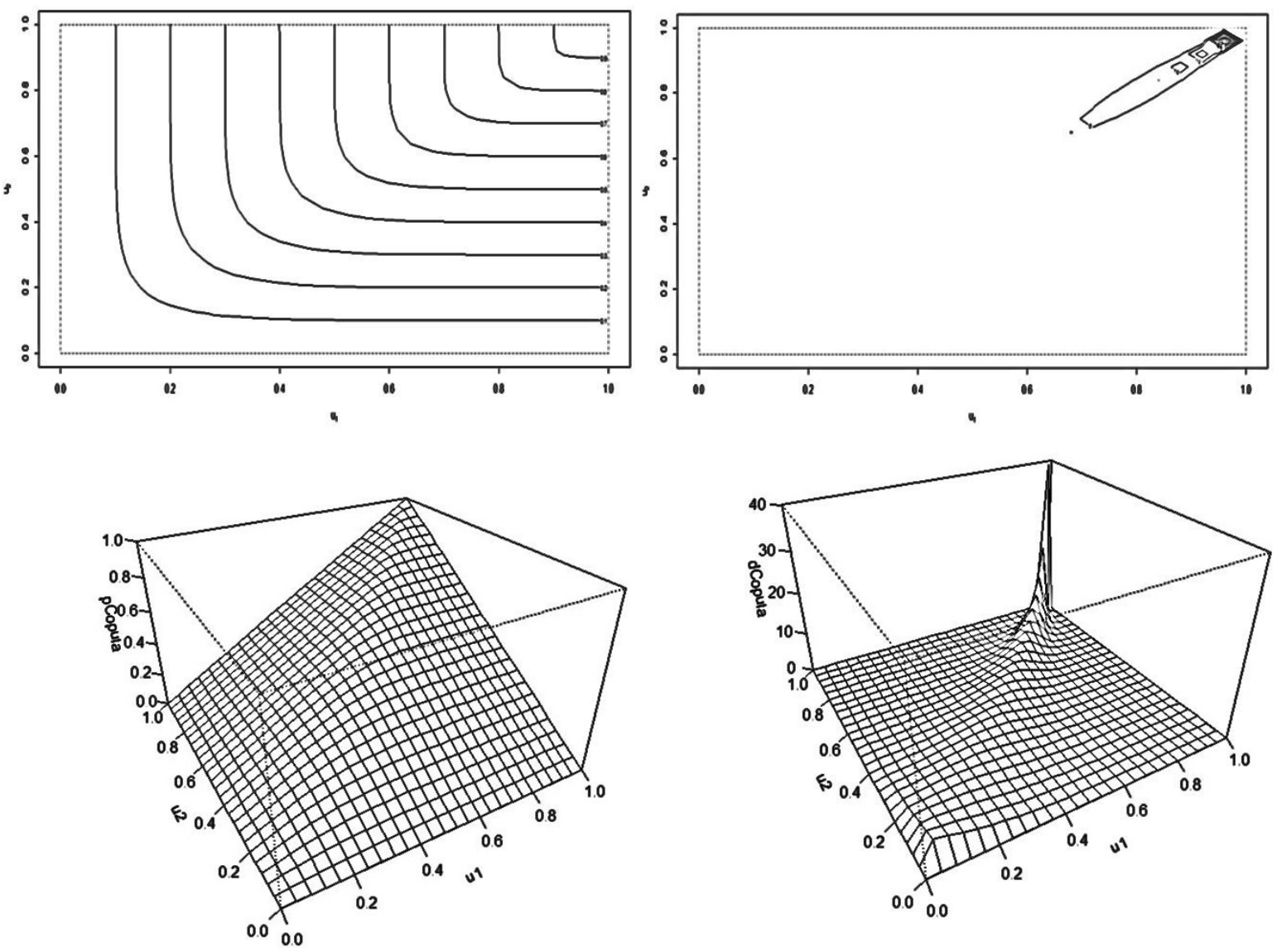

Figure 7: Respectively two and three dimension for Joe Copula family $\theta=6,8142$, probability and distribution function

\subsection{Plackett Copula Family and Spearman's Rho Dependency Estimation}

The connection between Spearman's Rho and Plackett copula family is given table 1. From this statement the parameter $\hat{\theta}$ of Plackett copula family is estimated value of 78,7905. The expected frequencies of the $\hat{\theta}$ parameter shows in table 6. Chi-Square value of Plackett copula family calculates as 64,65556 . The critical value of Chi-Square test with 16 of freedom and 0,05 confidences level is 26,29623, accordingly Plackett copula family unsuitable for our data set.

Table 7: For Plackett Copula Family Expected Values 


\begin{tabular}{ccccccc} 
& $Y_{1}$ & $Y_{2}$ & $Y_{3}$ & $Y_{4}$ & $Y_{5}$ & Sum \\
\hline$X_{1}$ & 93 & 19 & 2 & 1 & 1 & 116 \\
$X_{2}$ & 19 & 72 & 21 & 3 & 0 & 115 \\
$X_{3}$ & 2 & 21 & 69 & 21 & 3 & 116 \\
$X_{4}$ & 1 & 3 & 21 & 72 & 18 & 115 \\
$X_{5}$ & 1 & 0 & 3 & 18 & 94 & 116 \\
sum & 116 & 115 & 116 & 115 & 116 & 578 \\
\hline
\end{tabular}
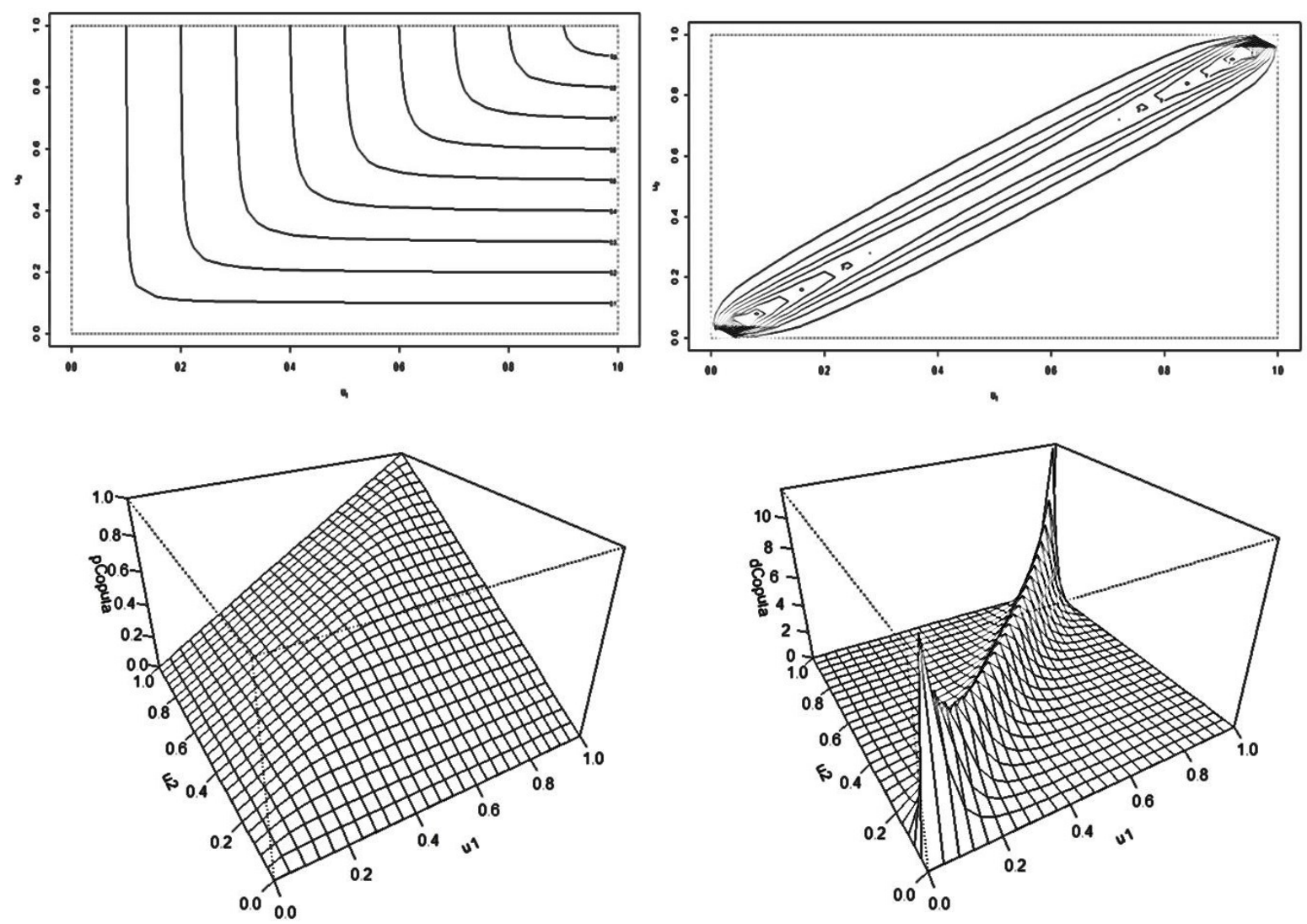

Figure 8: Respectively for two and three dimension Plackett Copula family $\theta=78,79507$ probability and distribution function

\section{Conclusion}

Nowadays, at the start of economy subject that has been mostly discussed comes topic of rate. If rate of dollar rise, it wait attempt of central bank. Aim of central bank is to provide price stability. Central Bank is not interesting adjustment policies but at the same time, extreme change of rate is become, it is study to remove this extreme change with foreign exchange purchase transaction. There is a relation between Deposit Rate and Exchange Rate of Dollars and for this relation is study about how strong. In this study degree of dependence between Deposit Rate and Exchange Rate of Dollars is obtained strong. The statistically significant copula algorithm can estimate. Dependency is modelling the Joe copula family $(\theta=6,8142)$ with Kendall Tau ( $\tau=0,751$ ). From this point of, The Joe copula is similar to Gumbel Copula, but the right tail positive dependence stronger. From this study, response of central bank on exchange Rate of Dollars is positively. Central Bank is response with purchase and sale on the exchange rate of dollars, when Extreme change of rate and statuses that it is break markets. Particularly, exchange rate fluctuations are cause development of our economic or in negativeness developments of derived from outside. In such cases, response of Central Bank is right positively. 
Conflict of interest: The authors declare they have no potential conflicts of interest with respect to the research, authorship, and/or publication of this article, and declare study has ethical permissions if required.

Acknowledgement: Contribution of Authors; AK MD: Concept, Design and Data Analysis, AK: Writing, Editing

\section{References}

1. A. Sklar. Fonctions de Repartition a $\mathrm{n}$ Dimensions et Leurs Marges. Publications de I'Institut de Statistique de I'University de Paris. 1959; 8: 229-231.

2. B. Schweitzer, E.F Wolff. On nonparametric measures of dependence for random variables. Annals of Statistics. 1981; 9: 879-885.

3 Cherubini, U., Luciano, E.. Value-at-Risk Trade-off and Capital Allocation with Copulas. Economic Notes. 2001; 30: $235-256$.

4. E.W. Frees, E.A. Valdez. Understanding relationships using copulas. North American Actuarial Journal. 1998; 2:1-25.

5. Genest C., J. MacKay.V. The joy of copulas: bivariate distributions with uniform marginal. The American Statisticien. 1986; 40: 280-283

6. Genest C. L.P. Rivest. Statistical inference procedures for bivariate Archimedean copulas. Journal of the American Statistical Association. 1993; 88 (423): 1034-1043.

7. Genest, C., Favre, A.-C. Everything You Always Wanted to Know About Copula Modelling butWere Afraid to Ask Journal of Hydrologic Engineering. 2006; 12: 347-368.

8. Genest, C., Gendron, M., Boudeau-Brien, M. The Adven of Copulas in Finance. The European Journal of Finance. 2009; 15: 609-618.
9. Malevergne, Y., Sornette, D. Testing the Gaussian Copula Hypothesis for Financial AssetsDependences. Quantitative Finance. 2003; 3 (4): 231-250.

10. Metin A, Çalık S. Copula Function and Application with Economic Data. Turkish Journal of Science and Technology. 2012; 7(2):199-204.

11. Naifar N. Modeling dependence structure with Archimedean copulas and applications to the iTraxx CDS index. Journal of Computational and Applied Mathematics. 2010; 235: 2459-2466

12. R. Nelsen. An Introduction to Copulas. Springer: Verlag; 1999.

13. Rosenberg, J., Schuermann, T. A General Approach to Integrated Risk Management withSkewed, Fat-tailed Risks. Journal of Financial Economics . 2006; 79: 569-614.

14. Shih, J.H., Louis, T.A. Inferences on the Association Parameter in Copula Models for Bivariate Survival Data. Biometrics. 1995; 51: 1384-1399.

15. Quesade-Molina, J.J. A generalization of an identity of Hoeffding and some applications. J.Ital.Stat.Soc. 1992; 3: $405-411$. 